\title{
Jejuno-jejunal intussusception in an adult - a rare presentation of abdominal pain in the Emergency Department
}

\section{Ahmed Hasan Yousef Al Zaabi}

Shaikh Shakhbout Medical City, Abu Dhabi

Jasmine Abdulla Al Janahi

Shaikh Shakhbout Medical City, Abu Dhabi

Salwa Najim Alremaithi

Shaikh Shakhbout Medical City, Abu Dhabi

\section{Balamurugan Rathinavelu}

Shaikh Shakhbout Medical City, Abu Dhabi

hasan qayyum ( $\nabla$ drqayyum@doctors.org.uk)

SSMC, Abu Dhabi https://orcid.org/0000-0002-7602-2981

\section{Case report}

Keywords: Emergency medicine, Gastroenterology, Small intestine, Intussusception, Surgery

Posted Date: June 22nd, 2021

DOI: https://doi.org/10.21203/rs.3.rs-629862/v1

License: (c) (i) This work is licensed under a Creative Commons Attribution 4.0 International License. Read Full License 


\section{Abstract \\ Background}

Abdominal pain is a common presentation to the emergency department (ED) and the differential diagnoses is broad. Intussusception is a more common diagnosis in children, with only $5 \%$ of cases reported in adults. $80-90 \%$ of adult intussusception is due to a well-defined lesion resulting in a lead point, whereas in children, most cases are idiopathic. Adult intussusception is also more commonly associated with malignancy, compared to children. In adults, malignancy is more common in intussusception involving the large bowel compared to intussusception in the small bowel.

\section{Case presentation}

We present a case of a 54-year-old lady who presented to our ED with abdominal pain and vomiting. She had multiple abdominal surgeries in the past. On examination, she had epigastric and peri-umblical tenderness. In view of her persistent abdominal pain that was refractory to analgesia, she had computed tomography (CT) of the abdomen which revealed a jejuno-jejunal intussusception and proximal small bowel obstruction. The patient had an urgent laparoscopy and small bowel resection of the intussusception segment was performed. No pathological lead point was identified on imaging or intraoperatively. The patient made a full recovery post operatively.

\section{Conclusion}

Our case report illustrates a rare diagnosis of abdominal pain and vomiting, presenting to the Emergency Department. With increasing accessibility to CT, most cases of adult intussusception are found incidentally on contrast CT of the abdomen and pelvis. While there is no consensus on management, it is more common for adult intussusception patients to have operative intervention, compared to childhood intussusception.

\section{Introduction}

The word 'intussusception' originates from modern Latin 'intus' (meaning within) and 'suscipere' (meaning to take up). It is the telescoping of the proximal portion of bowel (the intussusceptum) into the lumen of the contiguous bowel (the intussuscipiens).

It is a rare diagnosis accounting for $0.003-0.02 \%$ of all hospital admissions.[1][2] $5 \%$ of all intussusception cases occur in adults and they cause up to $5 \%$ of all adult bowel obstruction cases.[3][4] Initially described in the 17th century by Barbette of Amsterdam, in the 18th century, John Hunter described something similar except named in 'introsussuception'.[5][6] 
Diagnosis of adult intussusception is often tricky compared to children as clinical symptoms and signs are variable and non-specific. The onset is gradual, with most cases being subacute or chronic.[1][4][7] The classical clinical triad of abdominal pain, abdominal mass, and red currant jelly stool is rarely present in adults.[4][7][8] Abdominal pain is the most common symptom, seen in more than $70 \%$ of cases, others being nausea, vomiting, gastrointestinal bleeding, and abdominal distension.[1][2][6] Weight loss and constipation could be present and may suggest an underlying malignancy.[9]

In contrast to children, around $90 \%$ of adult intussusception cases have a well-defined, organic lesion resulting in a lead point.[1][4][10] This is located in the small bowel in the majority of cases.

Diagnosis is usually made intra-operatively in up to $50 \%$ cases,[2] although contrast enhanced CT of the abdomen is sufficiently characteristic to make a diagnosis based on radiological appearances.[4][6][11]

\section{Case Report}

A 54-year-old lady presented to our ED with severe abdominal pain, nausea, and vomiting. The pain was gradual in onset, located in the peri-umblical and epigastric area, and with no radiation. She denied any other symptoms.

She had multiple surgeries in the past including caesarean sections, the last one being 17 years ago, cholecystectomy 8 years ago, gastric bypass surgery 6 years ago, appendicectomy 3 years ago, and umblical hernia repair 2 years ago.

She has a history of hypertension and coronary artery disease controlled with medication. On our initial assessment, she was in severe abdominal pain with a pain score of $8 / 10$ requiring frequent doses of analgesia which reduced her pain score to $4 / 10$. Her vital signs were within normal limits except high blood pressure.

Systemic examination was within normal limits with the exception of the abdomen which was soft, nondistended with epigastric and peri-umblical tenderness. There were no palpable hernias. The abdomen was re-assessed after analgesia and similar findings were seen.

Differential diagnosis at this stage included bowel obstruction, possibly in the small bowel. This could have been related to an internal hernia or due to adhesions from previous surgery. Other differential diagnoses to exclude were myocardial infarction and pancreatitis.

Blood tests, a 12-lead electrocardiogram, chest X-ray and abdominal X-ray were performed which were all unremarkable. In view of her persistent abdominal pain, that remained refractory to analgesia, a decision was made to proceed with a contrast enhanced CT of the abdomen and pelvis. Oral and intravenous contrast was administered which demonstrated a jejuno-jejunal intussusception.

In Fig. 1, the intussusception can be seen in the CT coronal image of the abdomen and pelvis. This is demonstrated as a 'bowel within bowel' configuration with ingested contrast outlining the outer 
intussusceptum. Proximal small bowel obstruction is also seen.

The intussusception is also demonstrated as a round mass with intraluminal soft tissue and eccentric fat density, referred to as a target-like lesion in the CT axial image seen in Fig. 2. We were unable find a welldefined pathological lesion serving as a lead point based on CT.

This patient reported severe persistent abdominal pain requiring multiple doses of analgesia and fluid resuscitation. The surgical team were consulted in ED who assessed the patient and reviewed all investigations including CT images of abdomen and pelvis. In view of the patient's clinical condition in conjunction with the imaging reports, a decision was made to proceed for operative management The patient underwent an urgent diagnostic laparoscopy. This confirmed the pre-operative diagnosis of jejuno-jejunal intussusception located approximately $250 \mathrm{~cm}$ from the terminal ilium (Fig. 3). A small bowel resection containing the jejuno-jejunal intussusception segment was performed and a primary anastomosis fashioned. The immediate post-operative period was uneventful and the patient was treated with intravenous fluids and antibiotics. The patient was discharged from hospital after 3 days.

Subsequent histopathology of the resected bowel demonstrated focal edema in the mucosal and submucosal layers. No benign or malignant lesions were identified.

The patient was evaluated postoperatively in the hospital on day 8. She was well with no documented fever, abdominal pain, or vomiting. The wound site exam showed mild serous discharge with no signs of cellulitis and the abdomen was non-tender.

\section{Discussion}

Due to its variable presentation and often indolent course, the diagnosis of adult intussusception in ED requires a high index of suspicion.

Adult intussusception has been reported across ages, the highest numbers seen in the $30-50$ age group. The male: female incidence ratio is approximately 2:1.[1] Compared to children, malignancy is more commonly associated with intussusception in adults in up to $42-65 \%$ cases.[5][7]

Abdominal pain is the most common symptom, reported in more than $70 \%$ of cases. The onset is gradual with the average interval between onset and presentation being around 5 weeks, ranging from 1 day to 1 year.[1] Around half of all cases have a pre-operative diagnosis of bowel obstruction.[1][5]

Where the small bowel is involved, patients usually present with colicky central abdominal pain and vomiting. Nausea and vomiting are also commonly reported in $40-60 \%$ of cases.

Diarrhoea is often seen in large bowel intussusception and can be associated with lower abdominal pain and blood/mucus in stool and has been reported in 2-29\% of cases.[1][10] 
There have been multiple classifications of adult intussusception described.[12][13][14] Dean's classification, from 1956, remains commonly used describing 4 types based on location.[13][15] Enteric intussusception is the commonest type seen in adults and originates in the small bowel. In ileocecal intussusception, the ileocecal valve becomes the lead point. In ileocolic intussusception, the ileum goes through the ileocaecal valve. In colocolic intussusception, the colon telescopes in a contiguous part of the colon.

Malignancy as a lead point, is more commonly associated with large bowel intussusception, in up to $80 \%$ of cases, with adenocarcinoma being the commonest malignant lesion seen. Other malignant lesions include lymphoma, lymphomasarcoma, and leiomyosarcoma. The rest are benign lesions like leiomyoma, endometriosis, and anastomosis.[4][16] In contrast, the majority of lead points in small bowel intussusception are benign in nature like lipomas, polyps, hemangiomas, Meckel's diverticulum, lymphoid hyperplasia and villous adenoma of the appendix. In small bowel intussusception, malignancy is seen in only $14-47 \%$ of cases.[4][15][16]

Laboratory investigations are not useful in establishing a diagnosis of intussusception. Often the first imaging requested when patients present with abdominal pain and vomiting is a plain abdominal Xray which has a low sensitivity to rule out bowel obstruction or intussusception.[11][16]

Contrary to children, in adults with suspected intussusception, contrast enema studies are rarely performed. Ultrasound has been used regularly to evaluate intussusception in children than in adults and with a skilled operator has a high sensitivity and specificity approaching $100 \%$ for intussusception in children. In adults, this is not the case.[11] Due to increasing accessibility and advances in CT technology, this is the imaging modality that more commonly diagnoses intussusception. Most cases are found incidentally on CT performed for non-specific abdominal pain or bowel obstruction.[11] Additional information around bowel viability and the etiology can also be identified with this modality.[11] Different appearances of intussusception are described on CT but the presence of a 'bowel-within-bowel' appearance is pathognomonic.[17] A target-like lesion is seen when the beam is at 90 degrees to the long axis of the intussusception segment. This appears as a round shadow with intraluminal soft tissue and eccentric fat density. The intussusception can also appear like a sausage-shaped mass when the beam is parallel to the long axis of the lesion. When oral contrast is administered, contrast coating of the outer opposing walls of the intussusceptum can also be seen. A kidney-like reniform appearance is also described.[11][16][17] Magnetic resonance imaging of the small bowel has similar accuracy to CT. However, it requires a stable patient to stay still for a longer period time and may not be as readily available as CT in the emergency department.[11]

The best practice for management of adult intussusception remains debatable. Well-designed therapeutic trials comparing surgical to non-surgical treatment in adults are lacking.(18) In contrast to children, in adults, most cases are managed operatively perhaps due to the higher likelihood of finding a pathological lesion.[17][18] Some studies suggest small bowel intussusception can be reduced without resection if an underlying benign lead point is suspected, a medically treated condition is found like inflammatory bowel 
disease, or if the surgery could result in short bowel syndrome.[8][19] It is recommended these cases have a follow up small bowel enteroscopy or capsule endoscopy to rule out an intraluminal lesion that may predispose to recurrent intussusception.[19] Indications for operative management in adult intussusception may include bowel obstruction, a palpable abdominal mass or a lead point identified on imaging, gastro-intestinal bleeding, constitutional symptoms of malignancy, and colo-colic or ileo-colic intussusception due to their greater association with malignancy.[19][20]

Another study reported surgery as the modality of treatment in patients with clinical features of acute abdomen, clinical or radiological signs of bowel obstruction, peritonism, septic shock, intussusception with a mass visible on CT scan, patients with a diagnosis of colonic or ileocolic intussusception (often associated with malignancy), even in the absence of acute abdomen.[21]

\section{Conclusion}

Intussusception is a rare diagnosis in adults with a variable and non-specific presentation. Abdominal pain is the commonest presenting feature and often the onset can be gradual.

Most cases are found incidentally on contrast CT of the abdomen and pelvis, or intra-operatively. Adult intussusception is more commonly associated with a well-defined pathological lesion acting as a lead point such as malignancy, compared to children. In adults, malignancy is more common in intussusception involving the large bowel compared to intussusception in the small bowel. While there is no consensus on management, it is more common for adult intussusception patients to have operative intervention, compared to childhood intussusception.

\section{Declarations}

\section{Acknowledgements}

None

\section{Author Contributions}

All authors contributed to manuscript drafting and editing. All authors read and approved the final draft. Dr Hasan Qayyum conceptualised the idea.

\section{Funding}

No honorarium, grant, or funding was received to produce this manuscript.

\section{Availability of data and materials}

Data sharing is not applicable to this article as no datasets were generated or analysed. 
Not required

\section{Consent for publication}

Written informed consent for publication of clinical details and images was obtained from the patient

\section{Competing interests}

The authors declare that they have no competing interests.

\section{References}

1. Azar T, Berger DL. Adult intussusception. Ann Surg. 1997;226:134-8.

2. Nagorney DM, Sarr MG, McILRATH DC. Surgical Management of Intussusception in the Adult: Ann Surg. 1981 Feb;193(2):230-6.

3. Dean DL, Ellis FH, Sauer WG. Intussusception in adults. Arch Surg. 1956;73:6-11.

4. Begos DG, Sandor A, Modlin IM. The diagnosis and management of adult intussusception. Am J Surg. 1997 Feb;173(2):88-94.

5. Zubaidi A, Al-Saif F, Silverman R. Adult Intussusception: A Retrospective Review. Dis Colon Rectum. 2006 Oct;49(10):1546-51.

6. Marinis A, Yiallourou A, Samanides L, Dafnios N, Anastasopoulos G, Vassiliou I, et al. Intussusception of the bowel in adults: A review. World J Gastroenterol. 2009;15(4):407.

7. WEiLBAECHER AD, Bolin JA. Hearn Dav. Intussusception in Adults.:5.

8. Takeuchi K, Tsuzuki Y, Ando T, Sekihara M, Hara T, Kori T, et al. The Diagnosis and Treatment of Adult Intussusception. J Clin Gastroenterol. 2003 Jan;36(1):18-21.

9. Potts J, Al Samaraee A, El-Hakeem A. Small bowel intussusception in adults. Ann R Coll Surg Engl. 2014 Jan;96(1):11-4.

10. Coleman MJ, Hugh TB, May RE, Jensen MJ. INTUSSUSCEPTION IN THE ADULT. ANZ J Surg. 1981 Apr;51(2):179-80.

11. Byrne AT, Goeghegan T, Govender P, Lyburn ID, Colhoun E, Torreggiani WC. The imaging of intussusception. Clin Radiol. 2005 Jan;60(1):39-46.

12. Donhauser JL, Kelly EC. Intussuception in the adult. Am J Surg. 1950;79:673-7.

13. Toso C, Erne M, Lenzlinger PM, Schmid J-F, Büchel H, Melcher G, et al. Intussusception as a cause of bowel obstruction in adults. SWISS MED WKLY::5.

14. Brayton D, Norris WJ. Intussusception in adults. Am J Surg. 1954;88:32-43.

15. McKay R. Ileocecal Intussusception in an Adult: the Laparoscopic Approach. 2006;4.

16. Gayer G, Zissin R, Apter S, Papa M, Hertz M. Adult intussusception-a CT diagnosis. Br J Radiol. 2002 Feb;75(890):185-90. 
17. Kim YH, Blake MA, Harisinghani MG, Archer-Arroyo K, Hahn PF, Pitman MB, et al. Adult Intestinal Intussusception: CT Appearances and Identification of a Causative Lead Point. RadioGraphics. 2006 May;26(3):733-44.

18. Hadid T, Elazzamy H, Kafri Z. Bowel Intussusception in Adults: Think Cancer! Case Rep Gastroenterol. 2020 Jan 20;14(1):27-33.

19. Shenoy S. Adult intussusception: A case series and review. World J GastrointestEndosc. 2017;9(5):220.

20. Erkan N, Hacıyanlı M, Yıldırım M, Sayhan H, Vardar E, Polat AF. Intussusception in adults: an unusual and challenging condition for surgeons. Int J Colorectal Dis. 2005 Sep;20(5):452-6.

21. Panzera F, Di Venere B, Rizzi M, et al. Bowel intussusception in adult: Prevalence, diagnostic tools and therapy. World J Methodol. 2021;11(3):81-7. doi:10.5662/wjm.v11.i3.81. Published 2021 May 20.

\section{Figures}




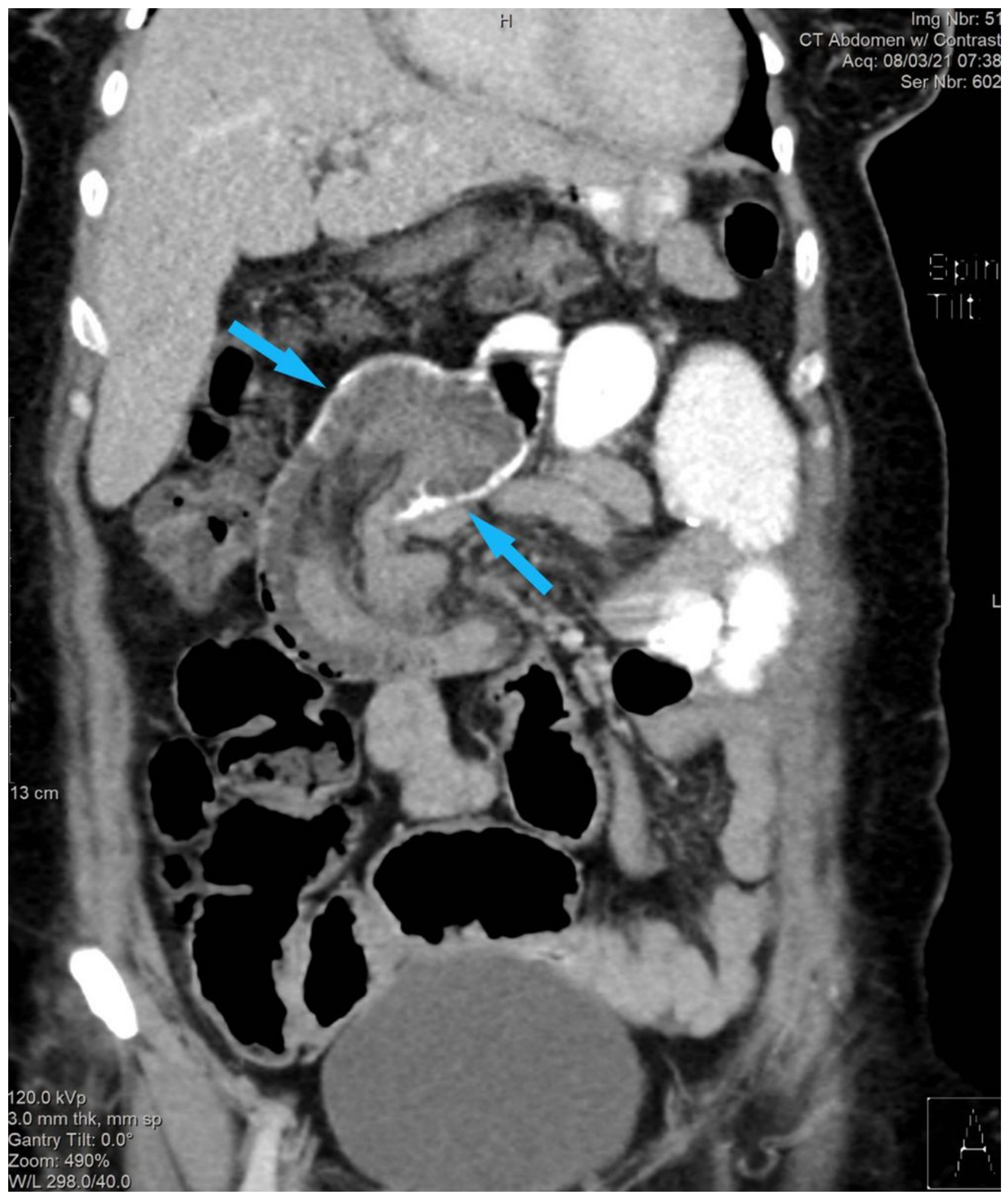

\section{Figure 1}

The intussusception can be seen in the contrast enhanced CT coronal image of the abdomen and pelvis. This demonstrates a 'bowel within bowel' configuration (blue arrow) with ingested contrast outlining the outer intussuscipiens. Proximal small bowel obstruction is also seen. 


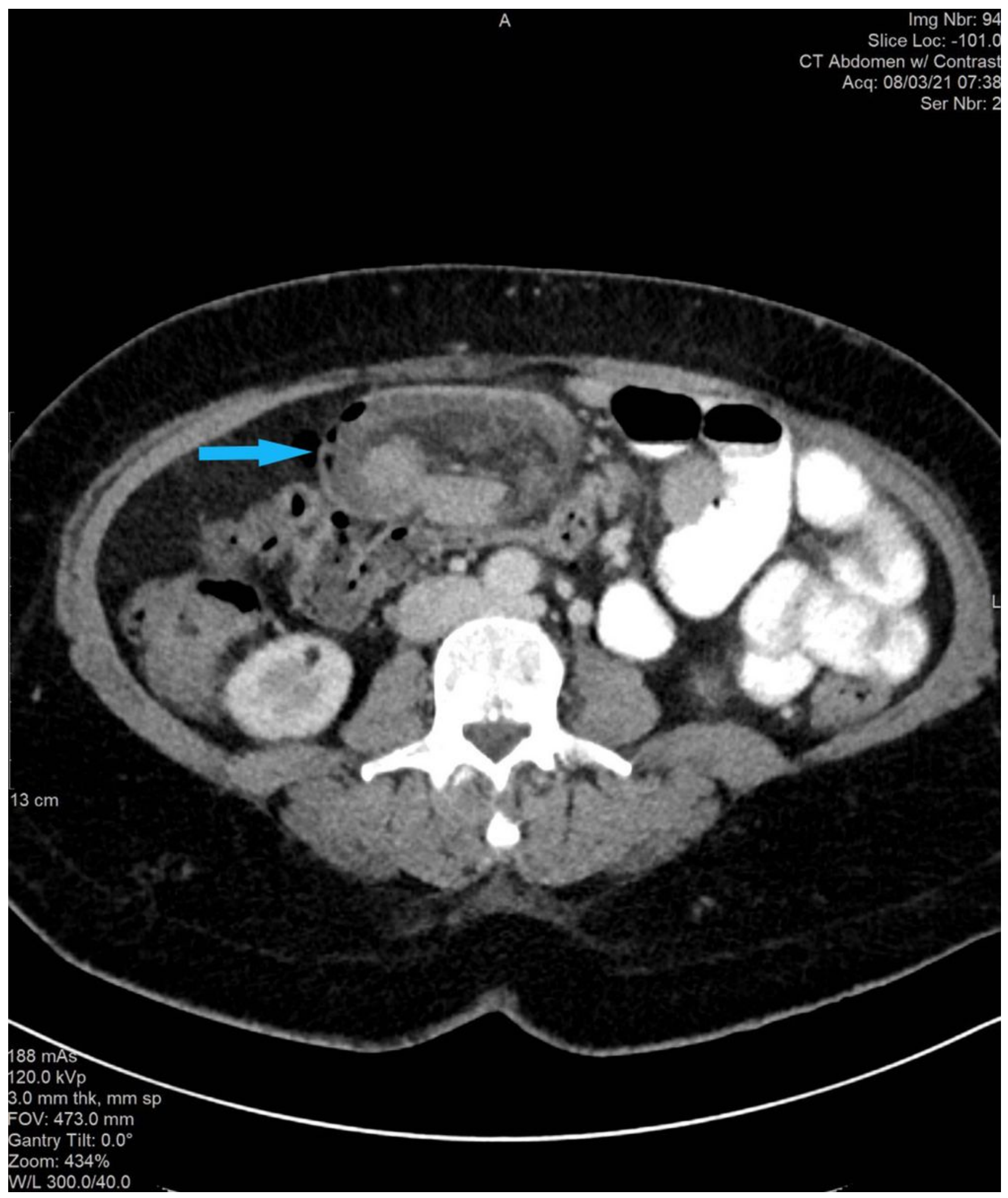

\section{Figure 2}

The intussusception is also demonstrated as a target-like lesion i.e.a round mass with intraluminal soft tissue and eccentric fat density (blue arrow) in the contrast enhanced CT axial image. 


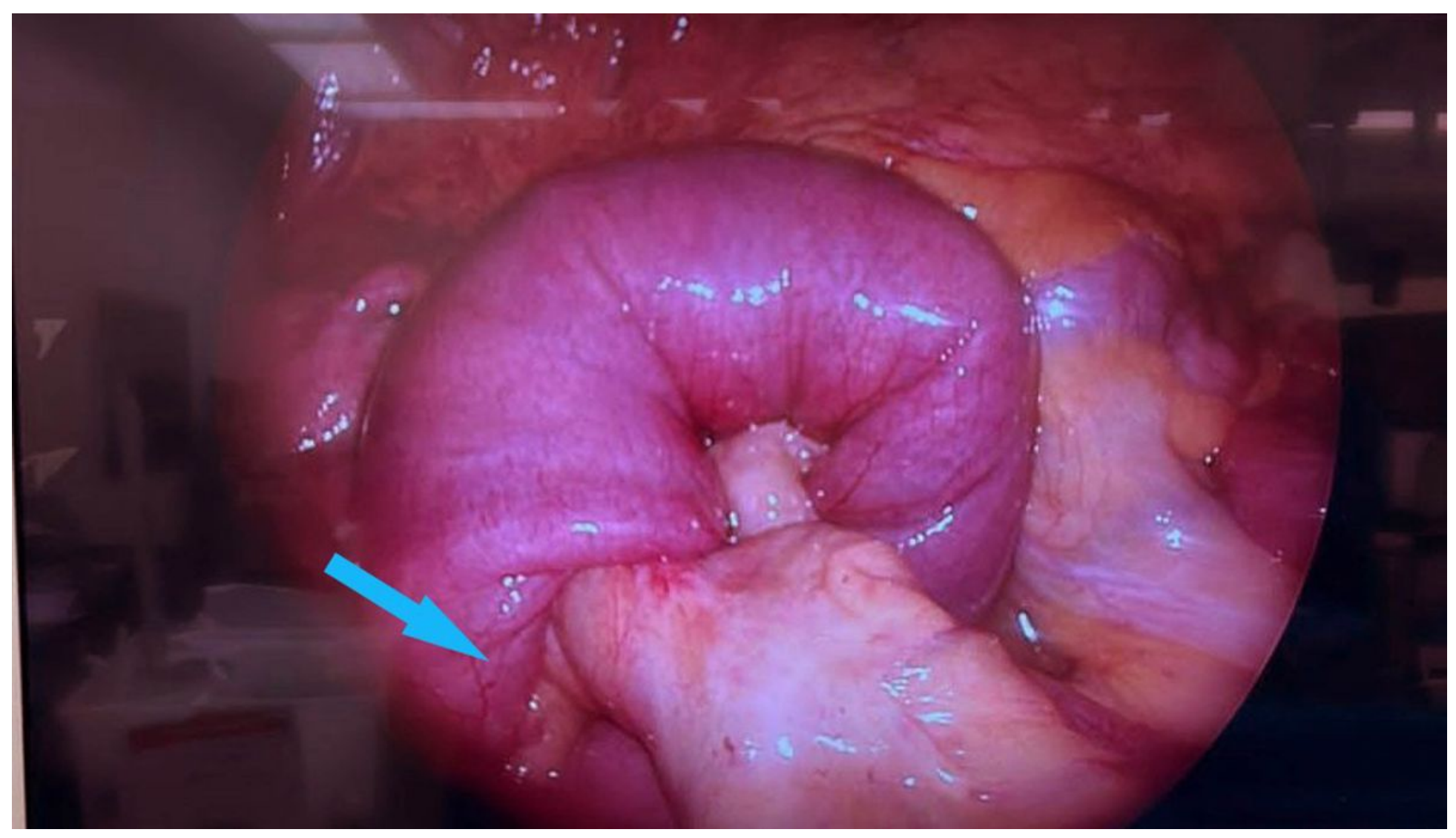

\section{Figure 3}

Intussusception segment on diagnostic laparoscopyshowing bowel (blue arrow) telescoping into a contiguous segment of bowel. 